\title{
Synthetic non-Abelian gauge fields and gravitomagnetic effects in tilted Dirac cone systems
}

\author{
T. Farajollahpour ${ }^{1}$ and S. A. Jafari $\circledast^{1,2, *}$ \\ ${ }^{1}$ Department of Physics, Sharif University of Technology, Tehran 11155-9161, Iran \\ ${ }^{2}$ Center of excellence for Complex Systems and Condensed Matter (CSCM), Sharif University of Technology, Tehran 1458889694, Iran
}

(Received 30 April 2020; revised manuscript received 6 June 2020; accepted 9 June 2020; published 29 June 2020)

\begin{abstract}
In planar tilted Dirac cone systems, the tilt parameter can be made space-dependent by either a perpendicular displacement field or by chemical substitution in certain systems. We show that the symmetric partial derivative of the tilt parameter generates non-Abelian synthetic gauge fields in these systems. The small velocity limit of these gauge forces corresponds to Rashba and Dresselhaus spin-orbit couplings. At the classical level, new forms of forces from conservative and Lorentz-type to (anti-)friction-like forces emerge from the effective spacetime structure in these materials. The velocity-dependent forces are odd with respect to tilt and therefore have opposite signs in the two valleys when the system is inversion symmetric. Furthermore, toggling the chemical potential between the valence and conduction bands by a gate voltage reverses the sign of the all these classical forces, which indicates these forces couple to the electric charge of the carriers. As such, these "gravitomagnetic" forces are natural extensions of the electric and magnetic forces that appear in the particular geometry of the tilted Dirac cone systems.
\end{abstract}

DOI: 10.1103/PhysRevResearch.2.023410

\section{INTRODUCTION}

In solid-state physics, the lattice breaks the Lorentz symmetry of the vacuum. But in certain lattices, such as the honeycomb lattice of graphene, the Lorentz symmetry emerges in a lower energy scale and with a velocity scale $v_{F}$, which is much smaller than the seed of light, ${ }^{1}$ with the following effective Hamiltonian:

$$
H_{D}=v_{F} \gamma^{0} \gamma^{i} p_{i}+m v_{F}^{2} \gamma^{0}
$$

where a possibly nonzero Dirac mass $m$ corresponds to the band gap. Here $d$ is the space dimensions, $i=1 \ldots d$ and $\gamma^{\mu}$ are Dirac matrices [1,2]. As usual Greek indices run from $0, \ldots, d$ with $\mu=0$ denoting the "time." The Dirac cone in the dispersion relation of the electronic degrees of freedom in Dirac solids at a deeper level can be attributed to an emergent effective Minkowski spacetime (at length scales much larger than the lattice constant), from which the Lorentz symmetry immediately and quite naturally follows.

But unlike the Lorentz symmetry of the vacuum of the standard model of particle physics, the emergent Lorentz symmetry of the condensed matter systems is not stringent and can be broken in a number of interesting ways. One way to break the Lorentz symmetry is to tilt the Dirac/Weyl cone in the spectrum. This can be done by adding a term $\hbar v_{F} \zeta^{i} p_{i}$

\footnotetext{
*akbar.jafari@gmail.com
}

Published by the American Physical Society under the terms of the Creative Commons Attribution 4.0 International license. Further distribution of this work must maintain attribution to the author(s) and the published article's title, journal citation, and DOI.

${ }^{1}$ In general, a mass term $\gamma^{0} m$ can also be included. proportional to unit matrix of appropriate dimension to the above Hamiltonian to give

$$
H_{D}=v_{F} \gamma^{0} \gamma^{i} p_{i}+m v_{F}^{2} \gamma^{0}+v_{F} \zeta^{i} p_{i}
$$

There are two approaches to study the Hamiltonian Eq. (2): (i) The first one is the standard solid-state approach to take this equation as the starting point and study its consequences and explore the effect of "tilt parameters" $\zeta \equiv \zeta^{i}$ in various physical properties. (ii) The second approach is to start from an effective spacetime structure specified by a metric $g_{\mu \nu}\left(\zeta^{i}\right)$ that reduces to the Minkowski structure as $\zeta^{i} \rightarrow 0$, namely $g_{\mu \nu}\left(\zeta^{i} \rightarrow 0\right)=\eta_{\mu \nu}=\operatorname{diag}\left(-1, \delta_{i j}\right)$, where $\delta_{i j}$ is the Kronecker $\delta$ function.

We start by providing a comprehensive introduction to relevant materials in various dimensions supporting the tilted cone dispersion, followed by a subsection introducing the spacetime structure of these materials. Those readers from gravitational physics background gan skip Sec. I A.

\section{A. Materials hosting tilted Dirac/Weyl cones}

Such tilted Dirac cone materials (TDCMs) and corresponding tilted Dirac fermions (TDFs) can exist in three (3D), two (2D), and even one (1D) space dimensions. For historical reasons, let us start the discussion with 2D TDFs. Historically, the layered organic compound $\alpha$-(BEDT-TTF $)_{2} \mathrm{I}_{3}$ was the first realization of tilted Dirac cone [3-8], which was discovered in Japan [9]. The Dirac structure of charge carriers in this compound is imprinted in their $\pi$ Berry phase inferred from their Landau quantization [10]. The tilt of the Dirac cone in organic compound can be inferred from interlayer magnetoresistance [11,12]. Landau levels were obtained from semiclassical quantization [13]. Quantum mechanically, from 
the solution in the presence of an in plane electric field, it was found that the valley degeneracy is lifted [14]. Magnetoplasmons in organic compound were studied by Sári et al. [15]. NMR measurements suggest that this compound is a strongly correlated TDF system [16] marked by three orders of magnitude enhancement of the Korringa ratio [17] and the reshaping of Dirac cone caused by strong Coulomb interactions in organic TDF system [18].

The list of TDFs in 2D is expanding rapidly. Starting from the above organic compound, substitution of iodine with halogens was examined, and it was found that replacement $\mathrm{I} \rightarrow \mathrm{F}$ can over-tilt the Dirac cone [19]. This is particularly important as it corresponds to transition from type-I to type-II Dirac fermions. Type-II 2D DFs are suggested by $a b$ initio calculations for quantum wells of $\mathrm{LaAlO}_{3} / \mathrm{LaNiO}_{3} / \mathrm{LaAlO}_{3}$ [20], where varying the number of $\mathrm{LaNiO}_{3}$ layers can shift the Dirac node. The surface of crystalline topological insulators [21] is also predicted to host TDFs. Furthermore, TDFs are proposed in the antiferromagnetic phase of the Iron-based superconductors as excitations above a spin density wave mean field state [22]. Angular resolved photoemission measurements of Varykhalov et al. which is also supported by $a b$ initio calculations, find a tilted Dirac cone on the metallic surface of $\mathrm{W}$ caused by Rashba spin-orbit interaction [23]. Ultra-low dissipation in the conductivity of $\mathrm{BaFe}_{2} \mathrm{As}_{2}$ was attributed to the tilted Dirac cone in its spectrum [24].

The above examples are 2D TDFs based on layered compounds. TDFs are also expected in purely $2 \mathrm{D}$, namely oneatom-thick compounds. First principle calculations suggest that partially hydrogenated graphene compound, $\mathrm{C}_{6} \mathrm{H}_{2}$ has a tilted Dirac cone (TDC) in its spectrum [25]. The left neighbor of Carbon in the periodic table of elements, namely, boron in 8Pmmn lattice structure is also predicted to host TDFs $[26,27]$. Group theory analysis of this structure of elemental boron suggests that the tilt of the Dirac cone in its spectrum can be tuned by a perpendicular electric field [28]

There has been many theory efforts to understand TDCMs. Kawarabayashi et al. [29] find a generalized chiral symmetry that protects the Dirac node in these systems. A generalized hopping model on honeycomb lattice supporting TDC is given by Kishigi et al. [30] as well as in quinoid-type graphene [13]. Initially motivated by the above organic compound, and later by the 2D 8Pmmn borophene, many physical properties of TDF systems are calculated. TDFs in 2D also exhibit the minimal conductivity phenomenon of upright Dirac fermions in graphene. In this case, $\sqrt{\sigma_{x x} \sigma_{y y}}$ approaches the same value as in graphene [31]. Proskurin et al. study longitudinal conductivity of 2D TDCMs in magnetic fields and find non trivial Landau levels transverse to tilt direction [32] and a divergent transverse conductivity [33]. Rostamzadeh et al. using Boltzmann and Kubo formulas obtain the ratio of the transverse and longitudinal conductivities diverges as $\sqrt{1-\zeta^{2}}$ [34], where $\zeta$ is the dimensionless quantity that determines the tilt. For typeI (II) TDFs one has $\zeta<1(>1)$. The effect of particle-hole asymmetry in the optical conductivity of TDFs was examined in [35]. Nishine et al. [36] find cusps in dynamical polarization that leads to new plasmon modes [37]. The analytical results of Jalali-Mola et al. also show a kink in the plasmon disper- sion, and an additional over-damped plasmon mode arising from the tilt $[38,39]$. The tilted is a very essential element in a minimal model that is able to generate a finite quadruple moment $\mathcal{Q}_{i j}$ [40]. It has been suggested that the tilt of the Dirac cone influences the spin transport [41]. Anomalous heat flow driven by the tilt was found by Sengupta et al. [42] for 2D TDFs. Given the relevance of strong correlations in the organic TDF systems, the effect of disorder and Coulomb interactions was also studied in 2D DFs [43,44]. The role of Coulomb interactions in generating dynamical (excitonic) gap in two-dimensional TDCMs was investigated in [45] where it was found that the tilt suppresses the dynamical gap. Excitonic instability of the tilted Dirac fermions is also investigated by Ohki and coworkers [46]. Tilt plays important role when superconductivity is introduced: Faraei et al. find that both retro and specular Andreev reflected holes come closer to the normal to the interface upon increasing the tilt parameter and the Andreev reflection becomes perfectly perpendicular when $\zeta \rightarrow 1$ [47]. Furthermore in SNS junctions based on 2D TDFs, the Andreev mode that propagates along the channel will acquire an electric charge when the tilt parameter is nonzero [48]. The pairing correlations are in general enhanced upon approaching the limit $\zeta=1$ that separates the type-I and type-II Dirac/Weyl fermions [49].

In three dimensions the tilted or type-II Weyl fermions in $\mathrm{WTe}_{2}$ were originally predicted and noticed as their Weyl node was protected as the meeting point of electron and hole pockets [50]. This prediction was soon confirmed in experiment [51-53]. Later on $\mathrm{MoTe}_{2}$ was also predicted [54] and confirmed [55-59] to be a tilted Weyl material. The Fermi arcs in $\mathrm{WTe}_{2}$ compound were observed in [52]. Later on, also type-II Dirac fermions were predicted in $\mathrm{PtSe}_{2}, \mathrm{PtTe}_{2}, \mathrm{PdTe}_{2}$ and $\mathrm{PtBi}_{2}$ family of dichalcogenides [60]. Single crystals of bulk $\mathrm{PtSe}_{2}$ were grown and evidence for type-II Dirac cone was found in Ref. [61]. Fei et al. [62] found nontrivial Berry phase in layered type-II Dirac fermion in $\mathrm{PdTe}_{2}$ crystals. They also found that Pt alloying of $\mathrm{IrTe}_{2}$ can shift the node to approach the type-II Dirac semimetal [63]. The $\mathrm{PdTe}_{2}$ compounds hosts both superconductivity and type-II Dirac fermions [64]. ARPES evidence for Lorentz violating type-II Dirac fermions was found in bulk $\mathrm{PtTe}_{2}$ [65]. The inverse Perovskite compound $\mathrm{Ca}_{3} \mathrm{PbO}$ also hosts 3D TDFs [66]. Subsulfide $\operatorname{Ir}_{2} \operatorname{In}_{8} \mathrm{~S}$ is also shown to host type-II 3D Dirac fermions [67].

On the theory side, Trescher and coworkers found that a simple anisotropy affects the conductance, but not the Fano factor, while the tilt affects both Fano factor and conductance [68]. Effect of disorder was also found to be enhanced in three-dimensional tilted Weyl materials [69]. Landau quantization of 3D type-I and type-II Weyl semimetals was investigated by Tchoumakov et al. [70]. Optical signature of the tilt in type-I and type-II Weyl semimetals was calculated by Carbotte [71]. In effect of disorder and Coulomb interactions was studied using renormalization group method in 3D TDFs [72]. It was found that the disorder enhances the effective tilt [72]. Furthermore, the tilt was found to give rise to intrinsic anomalous Hall conductivity [73].

Finally, regarding possible materials for 1D TDFs, it has been suggested that the sodium termination of zigzag edges in 
graphene nano-ribbons can give rise to $1 \mathrm{D}$ tilted Dirac cone spectrum [74].

\section{B. Tilted Dirac/Weyl materials as new spacetime structure in the solid state}

The list of materials exhibiting tilted Dirac/Weyl fermions is being expanded in both theory and experimental fronts in $d=1,2,3$ dimensions. There are two approaches to such systems. (i) The first approach is to take the tilt in the energy dispersion as granted. Then one can write an effective Hamiltonian compatible with the tilt and study its consequences. (ii) The second line of thought is to attribute the tilt in the dispersion relation to a new spacetime structure. This approach is pioneered by Volovik in three-dimensional Weyl semimetals and is followed by others. The later approach enjoys a covariant mathematical structure and necessitates the use of the geometric language of general relativity. Therefore, the powerful language of geometry can make certain phenomena more manifest and/or transparent by expressing the physics in a covariant and mathematically neat form. In this way, the plethora of phenomena associated with the structure of spacetime can be examined in a solid-state setting. In this part of the introduction we would like to elaborate on this aspect and review existing attempts in this direction: As pointed out for Dirac/Weyl materials with upright cone, the emergent structure of the spacetime felt by the electrons is the Minkowski spacetime. Tilting the cone-shaped dispersion spoils the Lorentz symmetry and hence the Minkowski structure of the emergent spacetime. Therefore, a valid and pertinent question would be, what is the new spacetime structure behind a tilted Dirac/Weyl cone spectrum?

This geometric line of thought in 3+1-dimensional Weyl semimetals is pioneered by Volovik [75,76]. Indeed the energy spectrum of Eq. (2) can be written as an invariant equation as $g^{\mu \nu} k_{\mu} k_{v}=m^{2}$, where $m$ can be either zero or nonzero Dirac mass, $k_{\mu}=(E, \boldsymbol{k})$ is the energy-momentum four-vector and $g_{\mu \nu}$ is given by the so called Painelevé-Gullstrand (PG) metric [28],

$$
d s^{2}=-v_{F}^{2} d t^{2}+\left(d \boldsymbol{r}-v_{F} \zeta d t\right)^{2} .
$$

In $3+1$ dimensions this metric can be brought to the standard Schwarzschild format [77] which admits a black-hole horizon [78]. The explicit form of the above metric in $3+1$ dimensions is

$$
g_{\mu \nu}=\left[\begin{array}{cccc}
\zeta^{2}-1 & -\zeta_{x} & -\zeta_{y} & -\zeta_{z} \\
-\zeta_{x} & 1 & 0 & 0 \\
-\zeta_{y} & 0 & 1 & 0 \\
-\zeta_{z} & 0 & 0 & 1
\end{array}\right]
$$

where $\zeta^{2}=|\zeta|^{2}=\zeta_{x}^{2}+\zeta_{y}^{2}+\zeta_{z}^{2}$. In $2+1$ dimensions, the last column and row of the above matrix will be omitted. Note that embedding the two-dimensional graphene and deforming it by strain, possible Lobachevsky space geometry can be constructed $[79,80]$. But the important difference of graphene metric with the $2+1 \mathrm{D}$ version of metric Eq. (4) is that in graphene only the spatial components $g_{i j}$ can be influenced by strain, while in tilted Dirac/Weyl materials the $g^{0 j}$ components mixing space and time are subject to change.
Furthermore, strain-induced changes are typically very small effects. Formally, the PG metric is basically a combination of a Galilean boost on a Minkowski metric and can be realized in rotating frames which can lead to interesting coupling between (quantum mechanical) spin and mechanical rotation $[81,82]$.

Although the tilting deformation of the Dirac theory destroys the standard Lorentz symmetry, but even for a uniform tilt parameter (i.e., a tilt parameter independent of spacetime coordinates), a deformed version of the Lorentz symmetry appears [83]. Such a modified Lorentz symmetry can be obtained as isometry of the deformed Minkowski spacetime via standard mathematical procedure [83]. Therefore, this symmetry can be attributed to a new spacetime structure. Indeed, the polarization function of tilted Dirac cone systems was shown to acquire a covariant form in the deformed Minkowski spacetime that is precisely defined by metric Eq. (4) [84].

Ojanen and coworkers propose that spatially varying timereversal (TR) and inversion (I) breaking sources in Weyl semimetals are equivalent to a curved spacetime for chiral fermions [85]. Such structures give rise to synthetic gauge fields. The present authors have proposed that in $2+1$ dimensions, the 2D spatial atomic arrangements allow to tune the geometry of the spacetime by electric fields [28]. Unlike strain induced changes in the metric of the spacetime, the changes introduced by TR or I breaking agents in (particularly 2D materials) is not a small effect. These ideas are further extended to meta-materials based on Weyl semimetals by Ojanen and coworkers [86] to design the structure of the spacetime.

In this paper we are interested in a $2 \mathrm{D}$ material hosting a tilted $2+1$-dimensional tilted Dirac cone. There are two Dirac cones that in the inversion symmetric case are described by two tilted Dirac cones with opposite tilt parameters $\zeta$ and $-\zeta$. Otherwise, their tilt parameters are arbitrary. In this paper we will study in detail the consequences of the spacetimedependent tilt parameter $\zeta$ and will show the emergence of non-Abelian gauge fields that in the nonrelativistic limit can be interpreted as geometry induced spin-orbit couplings. We further obtain the effect of curvature on the semi-classical motion (geodesics) and show that the tilt parameter gives rise to new forces which will be required in appropriate extensions of the Boltzmann equation in such spacetimes.

\section{NON-ABELIAN GAUGE THEORY IN TILTED DIRAC CONE MATERIALS}

In this paper we will be interested in the Dirac materials with tilted conic spectrum in two space dimensions. The metric of the resulting $2+1$-dimensional spacetime with tilt parameters $\zeta=\left(\zeta_{x}, \zeta_{y}\right)$ is given by

$$
\begin{aligned}
& g_{\mu \nu}=\left[\begin{array}{ccc}
\zeta^{2}-1 & -\zeta_{x} & -\zeta_{y} \\
-\zeta_{x} & 1 & 0 \\
-\zeta_{y} & 0 & 1
\end{array}\right], \\
& g^{\mu \nu}=\left[g_{\mu \nu}\right]^{-1}=\left[\begin{array}{ccc}
-1 & -\zeta_{x} & -\zeta_{y} \\
-\zeta_{x} & 1-\zeta_{x}^{2} & -\zeta_{x} \zeta_{y} \\
-\zeta_{y} & -\zeta_{x} \zeta_{y} & 1-\zeta_{y}^{2}
\end{array}\right]
\end{aligned}
$$


where $\zeta^{2}=\zeta_{x}^{2}+\zeta_{y}^{2}$. The components $\zeta_{x} \equiv \zeta_{1}$ and $\zeta_{y} \equiv \zeta_{2}$ of the tilt are assumed to have arbitrary functional dependence on the space coordinates $(x, y)$ inside the material. As a concrete example of how to generate such a space dependence in the tilt parameter $\zeta$, we have previously shown in 8 pmmn borophene, that an external displacement field perpendicular to the $2 \mathrm{D}$ material couples to electronic degrees of freedom in such a way that it controls the tilt parameters $\zeta$ [28]. Therefore, a given space-dependent profile of perpendicular electric field will imprint a corresponding profile on the metric. This will in general amount to electric-field control of the geometry of the spacetime. Another possible rout based on organic compounds would be the replacement of iodine with halogens [19] in a space-dependent manner.

Before studying the effect of a generic spacetimedependent entries $\zeta$ in Eq. (5) on the physical properties of TDCMs, let us recall the physics of strain in graphene. The strain in 2D materials can be formalized in terms of space-dependent metric entries. The resulting metric induced from a 3D Euclidean space to describe a deformed graphene gives rise to a curvature [87]. The Gaussian curvature of the deformed graphene will be equivalent to an effective (pseudo) magnetic field. ${ }^{2}$ Therefore, it is tempting to think that in the spacetime Eq. (5) of the TDCMs too, the role of spatial variation in $\zeta$ parameters will mimic an effective magnetic field. However, as we will show in this section, allowing $\zeta\left(x^{\mu}\right)$ to depend on spacetime coordinates, will generate non-Abelian gauge fields which in the nonrelativistic limit correspond to various forms of spin-orbit interactions. This is unlike the Abelian (pseudo)gauge fields arising from strain in graphene.

To understand the source of this difference, please note that in the case of strain fields in typical 2D materials, the strain field affects the spatial components $g_{i j}$ of the resulting metric. Due to the Minkowski nature of the parent graphene Hamiltonian, the strain field can not induce $g_{\mu 0}$ entries (offdiagonal entries mixing space and time). However, in the present case, the structure of the emergent metric Eq. (5) of the TDCMs is such that, allowing the $\zeta$ to vary in space, can only modify the off-diagonal components $g_{\mu 0}$. The space part, $g_{i j}$ remains totally diagonal. As such, as long as there is no strain field to generate off-diagonal entries in the spatial part, $g_{i j}$ of Eq. (5), we will not have any effective pseudo magnetic field corresponding to $U(1)$ gauge fields. Then the question is, what type of forces are generated in this case?

To answer this question, we will need a brief reminder from standard geometry knowledge (see any standard textbook on general relativity, e.g., Refs. $[78,88,89])$. For a spacetime with arbitrary metric $g_{\mu \nu}$, the Christoffel symbols defined by [90],

$$
\Gamma_{\mu \nu}^{\rho}=\frac{1}{2} g^{\rho \sigma}\left(\partial_{\mu} g_{\nu \sigma}+\partial_{\nu} g_{\mu \sigma}-\partial_{\sigma} g_{\mu \nu}\right),
$$

are the essential entities that allow us to (i) construct "covariant" derivative, (ii) construct equations of motions of particles (geodesics), (iii) to obtain the curvature tensor $[78,88,89]$.

\footnotetext{
${ }^{2}$ Here pseudo means that the sign of such magnetic field is opposite the two valleys.
}

\section{Emergent non-Abelian gauge fields}

As we pointed out, the first use of Christoffel symbols Eq. (6) is to construct covariant derivatives. Assume that a vector is specified by its components $V^{\mu}\left(\right.$ or $V_{\mu}$ ). The covariant derivative is given by

$\nabla_{\nu} V^{\mu} \equiv V_{; \nu}^{\mu}=V_{, \nu}^{\mu}+\Gamma_{\lambda \nu}^{\mu} V^{\lambda}, \nabla_{\nu} V_{\mu} \equiv V_{\mu ; \nu}=V_{\mu, \nu}-\Gamma_{\mu \nu}^{\lambda} V_{\lambda}$,

where $V_{v}^{\mu} \equiv \partial_{\nu} V^{\mu}$ is the partial derivative. Let us see where do we need to use these derivatives. The Dirac equation in general is expressed by first-order derivative $\left(\gamma^{\mu} \partial_{\mu}+m\right) \psi=$ 0 . In an arbitrary geometry, the curvature of spacetime requires to take the derivative of the spinor $\psi$ in a covariant form. This amounts to replacement $\gamma^{\mu} \partial_{\mu} \rightarrow \gamma^{a} e_{a}^{\mu}\left(\partial_{\mu}+\Omega_{\mu}\right)$ where the $\Omega_{\mu}$ is called the "spin" connection, as it needed to take covariant derivative of an "spinor" $[78,88,91,92]$. When the parameters $\zeta$ do not depend on space coordinate, the spin connection is $\Omega_{\mu}=0$ (for details see Appendix B). The frame fields $e_{a}^{\mu}$ will be required to change the basis in a way that a locally flat Minkowski spacetime is obtained. When $\zeta$ becomes space-dependent, in addition to the basis change, one has to worry about the spin connection $\Omega_{\mu}$ which will be defined and computed below. The spin connection $\Omega_{\mu}$ is related to the covariant derivatives defined in Eq. (7) through the Christoffel symbols $\Gamma$ 's as follows: For a generic manifold defined by metric $g_{\mu \nu}$ with $\mu, v=0,1,2$, there is a locally flat (Minkowski) manifold defined by metric $\eta_{a b}(a, b=0,1,2)$ tangent to this manifold. Requiring the spacetime length element in both cases to be identical gives

$$
g_{\mu \nu}=\eta_{a b} e_{\mu}^{a} e_{\nu}^{b} \leftrightarrow g^{\mu \nu}=\eta^{a b} e_{a}^{\mu} e_{b}^{\nu},
$$

which defines the frame fields $e_{\mu}^{a}$ (in $3+1$ dimensions are called vierbeins). From these frame fields one can construct

$$
\omega_{\mu}^{a b}=e_{\lambda}^{a}(x) g^{\lambda \sigma}(x) \nabla_{\mu} e_{\sigma}^{b}(x),
$$

where the covariant derivative of the frame fields are

$$
\nabla_{\mu} e_{\sigma}^{a}=\partial_{\mu} e_{\sigma}^{a}-\Gamma_{\mu \sigma}^{\lambda} e_{\lambda}^{a} .
$$

Finally employing the generators $\Sigma_{a b}=\left[\gamma_{a}, \gamma_{b}\right] / 4$ of the Lorentz group, we can form the spin connection as

$$
\Omega_{\mu}=\frac{1}{2} \omega_{\mu}^{a b} \Sigma_{a b} .
$$

With the above quick reminder from geometry, we are now ready to compute the effect of arbitrary space dependence of $\zeta$ in 2+1-dimensional TDCMs. The first thing we need to do is to compute the Christoffel symbols $\Gamma$ 's. It is straightforward, but cumbersome ${ }^{3}$ to use Eq. (6) to explicitly obtain Eq. (A4). For details please see Appendix A. Applying the steps outlined above to the metric (5) after a long but straightforward algebra we obtain the components $\omega_{\mu}^{a b}$ of the spin connection. The nonzero components will be given by Eq. (B14), which can be compactly written as

$$
\omega_{i}^{0 j}=\alpha_{i}^{j}, \omega_{0}^{0 i}=-\alpha_{i}^{j} \zeta_{j}, \text { others }=0,
$$

\footnotetext{
${ }^{3}$ There are plenty of well established algebraic manipulation programs to calculate the above symbols and much more by just giving the functional form of the entries of the metric. For example, see GRQUICK, https://library.wolfram.com
} 
where the quantity $\alpha_{i}^{j} \equiv\left(\partial_{i} \zeta^{j}+\partial^{j} \zeta_{i}\right) / 2$ suggested by Eq. (B14) is the symmetric partial derivative of the tilt $\zeta{ }^{4}$

Equipped with these results, we are now ready to discuss the effect of the above spacetime structure in $2+1$ dimensions. To proceed further, let us choose the following representation for the Clifford algebra,

$$
\gamma^{0}=i \sigma_{z}, \gamma^{1}=\sigma_{y}, \gamma^{2}=-\sigma_{x} .
$$

Using the definition Eq. (11) of the spin connection and performing the summation over $a, b=0,1,2$, only nonzero $\omega_{\mu}^{a b}$ s contribute whereby we obtain

$$
\begin{aligned}
& \Omega_{0}=\frac{1}{2} \omega_{0}^{a b} \Sigma_{a b}=-\frac{1}{4}\left[\alpha_{1}^{j} \zeta_{j} \sigma_{x}+\alpha_{2}^{j} \zeta_{j} \sigma_{y}\right], \\
& \Omega_{i}=\frac{1}{2} \omega_{i}^{a b} \Sigma_{a b}=\frac{1}{4}\left[\alpha_{i}^{1} \sigma_{x}+\alpha_{i}^{2} \sigma_{y}\right] .
\end{aligned}
$$

Once the spin connections $\Omega_{\mu}$ are computed, one can readily construct the associated gauge fields $[91,92]$,

$$
\mathcal{A}_{a}=e_{a}^{\mu} \Omega_{\mu} .
$$

Using the explicit forms of the frame fields $e_{a}^{\mu}$ given in Appendix B, we obtain

$$
\begin{aligned}
& \mathcal{A}_{0}=e_{0}^{0} \Omega_{0}+e_{0}^{1} \Omega_{1}+e_{0}^{2} \Omega_{2}=0, \\
& \mathcal{A}_{1}=e_{1}^{0} \Omega_{0}+e_{1}^{1} \Omega_{1}+e_{1}^{2} \Omega_{2}=\Omega_{1}, \\
& \mathcal{A}_{2}=e_{2}^{0} \Omega_{0}+e_{2}^{1} \Omega_{1}+e_{2}^{2} \Omega_{2}=\Omega_{2} .
\end{aligned}
$$

This equation establishes that the space dependence of the tilt parameters $\zeta$ in metric Eq. (5), induces non-Abelian gauge fields given by Eq. (16).

\section{SPIN-ORBIT FROM GEOMETRY}

In the context of graphene, it is well known that strain fields induce $U(1)$ gauge fields [93-96]. As can be seen in Eq. (16), the components or the gauge field in the context of the spacetime structure Eq. (5) related to tilted Dirac materials have matrix structure which makes the non-Abelian gauge fields. In the context of solid-state systems, the Pauli matrices $\sigma_{i}$ can denote the real spin (such as the helical states of a topological insulator) or the pseudospins (as in the case of graphene). In this section we will show that the space dependence of $\zeta$ will generate a coupling between the orbital motion and the (pseudo-)spin $\sigma$. To develop an intuition for the meaning of such gauge fields, it is useful to consider a massive Dirac particle with $m \neq 0$ that allows to study the "nonrelativistic limit" of the underlying Dirac system. Such a mass term does not change the structure of the metric. The only modification arising from the mass term will appear in the right side of the dispersion relation which is given by the invariant form $g_{\mu \nu} k^{\mu} k^{\nu}=m^{2}$.

The meaning of the gauge structure Eq. (16) can be best understood by expanding the Dirac hyperbolas around the

\footnotetext{
${ }^{4}$ Note that $\zeta_{i}=\zeta^{i}$ are parameters of the spacetime metric, and the indices here are not raised or lowered by the metric itself. So the locations of indices in the right side of this equation does not matter and are just set to balance the location of the indices in the left side. This is manifest when comparing to Eq. (B14).
}

band minima and approximating them by parabolas. Therefore, in tilted Dirac/Weyl systems too, the states near the bottom of the conduction band (or top of the valence band) of the Dirac dispersion can be approximated by a parabolic band structure. In the context of the standard model of particle physics, this corresponds to nonrelativistic limit where velocities are much less than the speed of light (which in our case is replaced by the Fermi velocity, $v_{F}$ ). In this limit a Dirac-Foldy-Wouthuysen transformation reveals how the spin-orbit interaction emerges from the Dirac equation. In our case, the same procedure will lead to a rich structure of pseudospin-orbit coupling.

The nonrelativistic limit of the tilted Dirac equation will be given by

$$
\frac{1}{2 m}(\boldsymbol{p} \mathbf{1}-g \mathcal{A})^{2},
$$

where $\mathcal{A}$ is given by Eq. (16). Expanding the above expression, one generates three type of terms, (i) $\mathcal{A}$. $\mathcal{A}$, (ii) $\mathcal{A}$. $p$, and (iii) $\partial . \mathcal{A}$ terms. They are given by the following expressions:

$$
\begin{aligned}
\mathcal{A} \cdot \mathcal{A} & =\frac{1}{16}\left[\left(\alpha_{1}^{1}+\alpha_{1}^{2}\right)^{2}+\left(\alpha_{2}^{2}+\alpha_{1}^{2}\right)^{2}\right]=\sum_{i, j, k} \alpha_{i}^{j} \alpha_{i}^{k}, \\
2 \mathcal{A} \cdot \boldsymbol{p} & =\frac{1}{4}\left[\alpha_{1}^{1} p_{x} \sigma_{x}+\alpha_{2}^{2} p_{y} \sigma_{y}+\alpha_{1}^{2}\left(p_{x} \sigma_{y}+p_{y} \sigma_{x}\right)\right], \\
-i \boldsymbol{\partial} \cdot \mathcal{A} & =\frac{1}{4}\left[\left(\partial_{i} \alpha_{1}^{i}\right) \sigma_{x}+\left(\partial_{i} \alpha_{2}^{i}\right) \sigma_{y}\right] .
\end{aligned}
$$

The nonrelativistic limit of this Hamiltonian becomes

$$
\frac{1}{2 m}\left[\boldsymbol{p}^{2}+g \mathcal{A} \cdot \mathcal{A}+g \boldsymbol{d} . \boldsymbol{\sigma}+g \boldsymbol{b} . \boldsymbol{\sigma}\right],
$$

where

$$
d_{n}(\boldsymbol{p})=\alpha_{n}^{j} p_{j}, \quad b_{n}=-i \partial_{j} \alpha_{n}^{j},
$$

where $n, j$ run over the spatial indices 1,2 . This analysis clearly shows that the non-Abelian gauge potential, in the nonrelativistic limit corresponds to two contributions: (i) The $\boldsymbol{d}$ term that directly couples momentum and (pseudo)spin, is the emergent spin-orbit coupling that arises from the coordinate dependence of the $\zeta$ that parametrizes the metric Eq. (5). (ii) The $\boldsymbol{b}$ term $g \boldsymbol{b} . \boldsymbol{\sigma}$ is emergent Zeeman term. Note that, this must be distinguished from the curvature induced $U(1)$ field that couples to orbital motion of the electrons and can generate Landau quantization. This term being a Zeeman-like term, can only couple to the (pseudo-) spin degree of freedom. Note that both $\boldsymbol{d}$ and $\boldsymbol{b}$ terms are odd functions of $\boldsymbol{\zeta}$. Therefore, in an inversion symmetric Dirac material where one can toggle between the two valleys by $\zeta \rightarrow-\zeta$, this Zeeman-like term also changes sign and are therefore pseudo-Zeeman like terms.

Mathematically, the field $\zeta^{i} \equiv \zeta_{i}$ being a vector field either has a zero circulation, or nonzero circulation. When its circulation is zero, it can be written as

$$
\zeta_{i}=\partial_{i} \Phi .
$$

In this case, the important parameters $\alpha_{n}^{j}$ are given by, $\alpha_{n}^{j}=$ $\partial_{n} \partial_{j} \Phi$ which then for $d_{n}$ and $b_{n}$ gives

$$
d_{n}(\boldsymbol{p})=\partial_{n} \boldsymbol{p} \cdot \partial \Phi, \quad b_{n}=-i \partial_{n} \partial^{2} \Phi .
$$


For an inversion symmetric material, if one valley has a tilt parameter $\zeta$, then the other valley must have $-\zeta$. Therefore, it is reasonable to assume that in real space too, the field $\zeta$ arises from sinks and sources of equal charges $\pm Q .{ }^{5}$ Therefore, except for isolated pairs of points, the condition $\partial \cdot \zeta=0$ is satisfied. The implication of this condition on $\Phi$ is, $\partial^{2} \Phi=$ $\pm Q \delta\left(\boldsymbol{r}-\boldsymbol{r}_{ \pm}\right)$. Choosing a linear combination of Harmonic functions to satisfy the boundary condition $\partial \Phi(\boldsymbol{r} \rightarrow \infty)=$ $\zeta_{\infty}$ (assuming a flat spacetime profile specified by $\zeta_{\infty}$ ) gives $\Phi=\zeta_{\infty} . \boldsymbol{r}+Q \ln \left(\left|\boldsymbol{r}-\boldsymbol{r}_{ \pm}\right|\right)$. The charges $\pm Q$ are integer topological charges of the field $\zeta$. Nonzero values of $Q$ when inserted in Eq. (22) give a singular contribution $\partial_{n} Q \delta\left(\boldsymbol{r}-\boldsymbol{r}_{ \pm}\right)$. Therefore, the topological index $Q$ governing $b_{n}$, can not be nonzero. The only remaining term $\zeta_{\infty} . r$ when inserted in Eq. (22) will give zero. The conclusion is that, if $\partial \times \zeta=$ 0 , both the spin-orbit coupling and the curvature induced Zeeman fields vanish.

Now let us consider the second possibility, namely a nonzero $\boldsymbol{\partial} \times \boldsymbol{\zeta}$. A general enough choice of $\boldsymbol{\zeta}=\rho f(\rho) \hat{\varphi}$ where $\hat{\varphi}$ is the unit vector in cylindrical coordinate corresponding to azimuthal angle $\varphi$, and $\rho$ is the distance from the origin. This choice gives

$$
\boldsymbol{\partial} \times \boldsymbol{\zeta}=\frac{1}{\rho} \frac{\partial}{\partial \rho}\left[\rho^{2} f\right] \hat{z} .
$$

In this case, the important parameters $\alpha_{n}^{j}$ are given by

$$
\rho^{-1} f^{\prime}\left(x_{n} \varepsilon_{j \ell} x_{\ell}+x_{j} \varepsilon_{n \ell} x_{\ell}\right),
$$

where $\varepsilon_{12}=-\varepsilon_{21}=1$ and $\varepsilon_{11}=\varepsilon_{22}=0$ defines the LeviCivita symbol and $f^{\prime}=\partial_{\rho} f$. Inserting the above result in Eq. (20) gives

$$
\begin{aligned}
d_{1} & =f^{\prime} \rho\left(p_{x} \sin 2 \varphi+p_{y} \cos 2 \varphi\right) / 2, \\
d_{2} & =f^{\prime} \rho\left(p_{x} \cos 2 \varphi-p_{y} \sin 2 \varphi\right) / 2, \\
b_{1} & =(-i) \rho \sin \varphi\left(\rho g^{\prime} \sin ^{2} \varphi-3 g\right), \\
b_{2} & =(-i) \rho \cos \varphi\left(-\rho g^{\prime} \cos ^{2} \varphi-3 g\right),
\end{aligned}
$$

where we define $g=f^{\prime} / \rho$. Adopting a representation for the Clifford algebra that differs by the one in Eq. (13) in $\sigma_{x} \leftrightarrow \sigma_{y}$, for the spin-orbit coupling term we obtain

$$
f^{\prime} \rho(\cos 2 \varphi \boldsymbol{\sigma} \cdot \boldsymbol{p}-\sin 2 \varphi \boldsymbol{\sigma} \times \boldsymbol{p}) / 2 .
$$

An important feature of the above spin-orbit coupling is its highly anisotropic nature. The nice-looking terms $\boldsymbol{\sigma} \cdot \boldsymbol{p}$ is actually the Dresselhaus spin-orbit coupling. To see this one can transform back to the old representation Eq. (13). The second term, $\boldsymbol{\sigma} \times \boldsymbol{p}$ in the new representation is clearly seen to be a Rashba spin-orbit coupling. Therefore, the space dependence of the tilt parameter $\zeta$, whose circulation is nonzero (determined by $f$ ) gives rise to Dresselhaus and Rashba spin-orbit couplings that are, (i) highly anisotropic, (ii) their existence depends on the value of $f^{\prime}$. Therefore, a $\rho$-independent $f$, despite giving rise to a nonzero constant circulation in Eq. (23) will have $f^{\prime}=0$ and hence both $\boldsymbol{d}$

\footnotetext{
${ }^{5}$ Note that here $Q$ is a topological charge associated with the configuration of the tilt field $\zeta$.
}
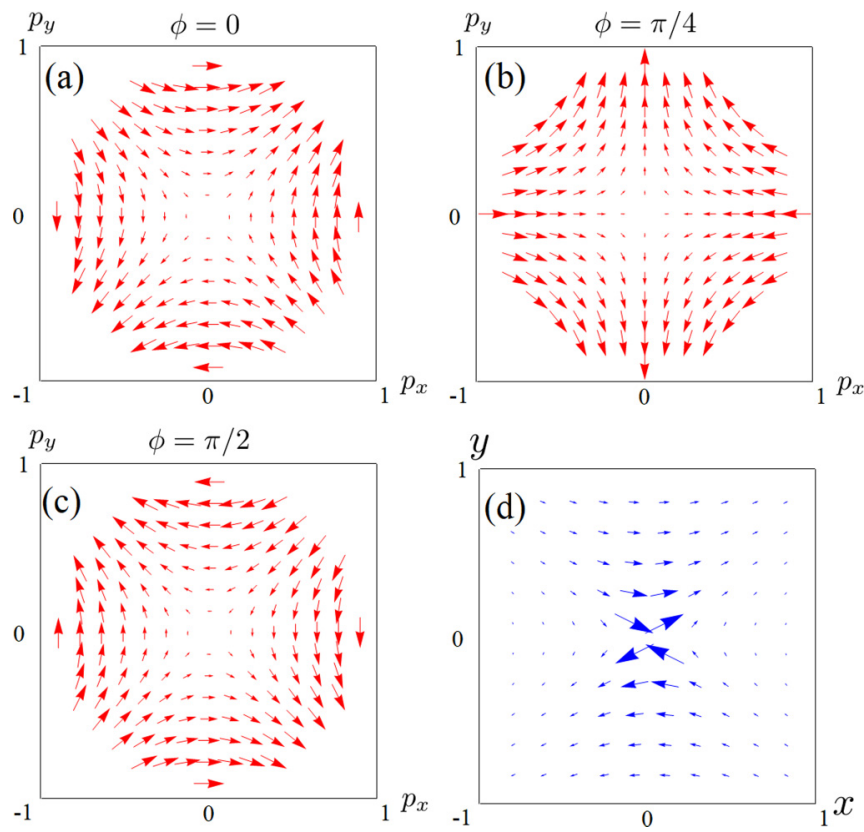

FIG. 1. Vector plot of spin-orbit coupling and pseudo-Zeeman field. (a-c) By choosing $f(\rho)=\ln \rho$ which is equivalent to $\zeta=$ $2 \rho \ln \rho \hat{\varphi}$, girves rise to $f^{\prime} \rho=2$ which eliminates the radial dependence of the spin-orbit coupling $\boldsymbol{d}$, plotted when $\phi=0, \pi / 4, \pi / 2$. (d) Choosing $g=2 \rho^{-2}$ leave a $1 / \rho$ radial dependence in the pseudoZeeman field $\boldsymbol{b}$.

and $\boldsymbol{b}$ vanish. This feature distinguishes the pseudo-Zeeman and pseudo-spin-orbit coupling that are generated by space dependence of the tilt parameter $\zeta$ from the other forms of spin-orbit coupling arising from rotation $[81,82,97]$.

As an example, choosing $f(\rho)=\ln \rho$ which is equivalent to $\zeta=2 \rho \ln \rho \hat{\varphi}$, gives rise to $f^{\prime} \rho=2$ which eliminates the radial dependence of the spin-orbit coupling $\boldsymbol{d}$ in Eq. (25). Correspondingly we have $g=2 \rho^{-2}$ which will leave a $1 / \rho$ radial dependence in the pseudo-Zeeman field $\boldsymbol{b}$. These features are plotted in Fig. 1. The above analysis in the small $\boldsymbol{p}$ reveals the structure of spin-orbit coupling (or pseudospinorbit coupling if $\sigma$ is not the real spin) arising from spacedependent tilt parameter $\zeta$. When the $\zeta$ that enters as an off-diagonal term mixing space and time is absent, as can be seen from Eq. (12), the $\omega_{\mu}^{i 0}$ components of the spin-connection vanish from which it follows that the non-Abelian gauge fields in Eq. (16) vanish. When the $\zeta$ term is introduced in tilted Dirac/Weyl systems and for whatever reason possess a nontrivial space dependence, the connections $\omega_{\mu}^{0 i}$ (which multiply the matrices $\gamma_{0} \gamma_{i}$ ) become nonzero and generate the non-Abelian gauge structure in Eq. (16). Allowing the spatial components of the metric to depend on space (driven, e.g., by strain), will generate the $\omega_{\mu}^{i j}$ components which are well known in the context of graphene and can only generate the $U(1)$ gauge structure, as they couple to $\gamma_{i} \gamma_{j}$ combination of Dirac matrices [93,94].

\section{CLASSICAL GEODESICS}

When the length scale of applied fields are much larger than the spread of the wave packets, and the wave packets 
themselves are larger than the lattice constant, the semiclassical Boltzmann transport can be used to study the electron dynamics. In tilted Dirac/Weyl materials too, such a regime does exist and it is therefore appropriate to study the classical geodesics in a generic background metric Eq. (5) with spacedependent $\zeta$. This will allow us to develop a feeling at the classical level to the nature of the forces that correspond to the the non-Abelian gauge structure Eq. (16).

It is a well known textbook fact that gravitational forces around a rotating source that only slightly deform the metric $\eta^{\mu \nu}$ of the flat spacetime, can be effectively described by forces that resemble the electromagnetic forces. These effects go under the name of gravitomagnetic effects. ${ }^{6}$ The effect of rotation goes into a weak off-diagonal element that mixes space and time. This feature is similar to our metric Eq. (5), except that (i) $\zeta$ in our case is not necessarily weak and (ii) its functional dependence on space coordinates $(x, y)$ can in principle be anything. Therefore, in this section we set out to understand the meaning of the space-dependent tilt parameter $\zeta$ in the metric Eq. (5) of the tilted Dirac/Weyl materials. We will follow the textbook approach of Ryder and will start by writing down the geodesics equations in the background metric Eq. (5) to identify the structure of the new forces that arise from spatial variation of $\zeta$ at the classical level. In the case of graphene where only the spatial components $g^{i j}$ of the metric are allowed to depend on space (due to strain field), it has been found that the curvature induced $U(1)$ forces, lead to spatial separation of the valley currents [98].

Our classical treatment in this section, parallels the quantum treatment of Sec. III as in both cases we consider the limit where a mass term is present that allows to approximate the Dirac hyperbola by a parabola. The geodesic equation is given by [88]

$$
\frac{d^{2} x^{\mu}}{d s^{2}}+\Gamma_{\nu \lambda}^{\mu} \frac{d x^{\nu}}{d s} \frac{d x^{\lambda}}{d s}=0,
$$

where $s$ is the proper time. In the nonrelativistic (or in gravitational jargon the "Newtonian") limit, $s \approx t$ and $d x^{0} / d s \approx$ $v_{F}$ and $d x^{i} / d s \approx v \ll v_{F}$. In this limit the acceleration $a^{i}=$ $d^{2} x^{i} / d t^{2}$ will become

$$
\begin{aligned}
a^{i}= & -\left(v_{F}^{2} \Gamma_{00}^{i}+2 v_{F} \Gamma_{0 k}^{i} v^{k}+\Gamma_{k m}^{i} v^{k} v^{m}\right) \\
& +\left(v_{F}^{2} \Gamma_{00}^{0}+2 v_{F} \Gamma_{0 k}^{0} v^{k}+\Gamma_{k m}^{0} v^{k} v^{m}\right) \frac{v^{i}}{v_{F}} .
\end{aligned}
$$

Performing the summations over $k, m$ the separate components $i=1,2$ of the acceleration can be organized in powers of $v / v_{F}$, where $v$ is the band velocity near the bottom of conduction band and $v_{F}$ is the asymptotic velocity of Dirac electrons at large momenta. The leading (zeroth) order is

$$
\boldsymbol{a}_{(0)}=\frac{v_{F}^{2}}{2}\left(\partial \zeta^{2}-\zeta \zeta \cdot \partial \zeta^{2}\right)
$$

The first term in this equation is a gradient term. If $\zeta^{2}$ can be interpreted as negative of an "electrostatic" potential, then

\footnotetext{
${ }^{6}$ See, e.g., chapter 6 of the Ryder's textbook [88].
}

the first term will correspond to the electric field arising from such term. There is, however, additional structure in the second term. When the variations of $\zeta$ are purely transverse, the longitudinal derivative $\boldsymbol{\zeta} \cdot \boldsymbol{\partial}$ will vanish. If the spatial profile of $\zeta^{2}$ generated by external fields is localized around some origin, ${ }^{7}$ and decays away from origin, then it corresponds to an inward acceleration if $m$ is positive (i.e., we for states near the bottom of conduction band). The gate voltage can be used to tune the chemical potential and therefore enables us to tune the Fermi level between the conduction and valence bands. This process will change the sign of $m$. Therefore, if the above term is attractive for electrons, then it will be repulsive for holes, and vice versa.

The first-order contribution is

$$
\begin{aligned}
a_{(1)}^{i}= & v_{F}\left(\vec{\zeta} \cdot \vec{\partial} \zeta^{2}-\partial_{i} \zeta_{\bar{i}}^{3}-\frac{1}{3} \partial_{i} \zeta_{i}^{3}\right) v^{i} \\
& +v_{F} v^{\bar{i}} \zeta_{i}\left(\vec{\zeta} \cdot \vec{\partial} \zeta_{\bar{i}}+\frac{1}{2} \partial_{i} \zeta^{2}\right)+v_{F}(\boldsymbol{v} \times \partial \times \zeta)^{i}
\end{aligned}
$$

where $i=1,2$ correspond to $\bar{i}=2,1$, respectively. Note that $i$ is the free index of the left side and there is no sum over $i$ on the right side.

For the inversion symmetric tilted Dirac materials the two valleys have opposite $\boldsymbol{\zeta}$. The velocity independent terms, $\boldsymbol{a}_{(0)}$ in Eq. (29) are even in $\zeta$. Therefore, they do not change upon $\zeta \rightarrow-\zeta$. Hence, these terms are the same in both valleys in such materials. The first-order terms in Eq. (31) are odd in $\zeta$. Therefore, these can be attributed to the pseudoforces in inversion symmetric system. Particularly, the third line in Eq. (31) is a Lorentz-type force provided $\zeta$ can be imagined as the spatial part of a vector potential such that $\partial \times \zeta$ would correspond to a pseudomagnetic field. Again by pseudo we mean that this field begin odd in $\zeta$, changes sign in the other valley. The first line resembles a "friction" force as the $i$ th component of the acceleration is proportional to $v^{i}$. Depending on the sign of the term in the parenthesis, this can be friction or antifriction! In the later case, this term will cause an increase in the velocity, until the Newtonian regime ceases to be valid. In analogy with the first line, one can think of the second line as a kind of transverse friction" force which is then expected to enhance the shear viscosity when the interactions are turned on to form an electron liquid.

To develop a feeling for the classical dynamics in the spacetime Eq. (5), let us consider the special case where $\zeta=$ $[h(x), 0]$ is varied only unidirectionally along the $\zeta$ direction. We will further assume that $h(x)=\zeta_{0} \tanh \left(\frac{x}{\lambda}\right)$. In this case, for the zeroth-order contribution we get,

$$
\begin{aligned}
a_{(0)}^{1} & =\frac{v_{F}^{2}}{2}\left(\partial_{x} \zeta^{2}-\zeta_{x}\left(\zeta_{x} \partial_{x}+\zeta_{y} \partial_{y}\right) \zeta^{2}\right) \\
& =\frac{v_{F}^{2}}{2} h(x) h^{\prime}(x)\left(1-h^{2}(x)\right) \\
& =\frac{v_{F}^{2}}{2} \frac{\zeta_{0}^{3}}{\lambda} \tanh \left(\frac{x}{\lambda}\right) \operatorname{sech}^{2}\left(\frac{x}{\lambda}\right)\left(1-\zeta_{0}^{2} \tanh ^{2}\left(\frac{x}{\lambda}\right)\right)
\end{aligned}
$$

\footnotetext{
${ }^{7}$ Perhaps this can be achieved by applying the electric field via a tip of thickness of several tens of nanometers.
} 
and

$$
a_{(0)}^{2}=\frac{v_{F}^{2}}{2}\left(\partial_{y} \zeta^{2}-\zeta_{y}\left(\zeta_{x} \partial_{x}+\zeta_{y} \partial_{y}\right) \zeta^{2}\right)=0
$$

and the first-order contribution is

$$
a_{(1)}^{1}=v_{F} h^{\prime}(x) h^{2}(x) v_{1}, \quad a_{(1)}^{2}=-v_{F} h^{\prime}(x) h^{2}(x) v_{2}
$$

which for our step-like function becomes,

$$
\left(a_{(1)}^{1}, a_{(1)}^{2}\right)=v_{F} \frac{\zeta_{0}^{3}}{\lambda} \operatorname{sech}^{2}\left(\frac{x}{\lambda}\right) \tanh ^{2}\left(\frac{x}{\lambda}\right)\left(v^{1},-v^{2}\right) .
$$

Note that in this section what we have calculated is the acceleration. To convert it to the force, one must note that it has to be multiplied by a mass term $m$. This term is positive for the states near the bottom of the conduction band. For those near the top of the valence band, this term (determining the parabola) is negative. Therefore, the sign of the above forces can be reversed by, e.g., gate doping and toggling the chemical potential between the valence to conduction bands. In this way, the sign of the force depends on the sign of charge carriers. Therefore the electric and magnetic forces emerging from the structure of the spacetime Eq. (5) reverse their signs by changing the "charge" of the carriers. As such, these forces can be regarded as a natural generalizatioins of electric and magnetic forces that arise from the geometry of the spacetime itself. The external electric or magnetic fields are assumed to be absent here. Therefore, in studying the effect of external electric and magnetic fields on the transport of electrons, one must in addition to external electric and magnetic fields worry about the forces that arise from the curved nature of spacetime Eq. (5).

For a fixed chemical potential corresponding to a given sign of the energy, the sign of $m$ is fixed. In that case, all the terms, including the term that resebles the Lorentz-force in the third line of Eq. (31) change sign. As such the classical Landau orbits for electrons in the two valleys have opposite directions. Therefore, this term (when strong enough) can generate valley-polarized edge currents. So the curvature engineering can in principle generate valley Hall effect. Even if the material is not perfectly inversion symmetric, the symmetric part of it (for which two valleys are related by $\zeta \rightarrow-\zeta$ ) is capable of generating valley polarized effects.

\section{DISCUSSIONS AND SUMMARY}

In this work we have investigated the effects arisging from spatial dependence in the tilt parameter $\zeta$ that defines the metric (5) in tilted Dirac cone materials. The essential quantity is $\alpha_{i}^{j}$ defined under Eq. (12). At quantum level this quantity gives rise to non-Abelian gauge fields. The meaning of such gauge fields becomes clear in the nonrelativistic limit pertinent the bottom of conduction or top of valence band states which corresponds to various forms of spinorbit coupling. This agrees with recent proposal by Shitade and coworkers on geometric spin-orbit coupling [97]. At the classical level, from geodesic equations one can infer various forms of forces that have no analogs in solid-state systems with Galilean spacetime structure. The sign of these forces can be changed by toggling between conduction and valence bands via a gate voltage and therefore for opposite charge carriers they have opposite signs. This means that these forces are natural extensions of electric and magnetic forces that arise from geometry (5). In studying transport properties of such systems, these forces are also expected to play role in addition to the external electric and magnetic fields acting on the charge carriers.

It is worth to reiterate why unlike the strain induced pseudo-gauge forces in graphene, here we have a non-Abelian gauge structure. The reason is that strain appears in spatial components $g_{i j}$ while the space dependence of the tilt in TDCMs appears through the off-diagonal entries $g_{0 \mu}$ that mix space and time coordinates. These two ways of modifying the $g_{\mu \nu}$ metric have two different physics. Therefore, strain can be used as additional control parameter to generate a pseudomagnetic field in addition to the spin-orbit interactions arising from the spatial variations of $g_{0 \mu}$ entries of the metric in TDCMs. Indeed generation of spin-orbit couplings by spatial variation of $\zeta$ seems quite plausible: In our previous work [28] we have found that the displacement field couples to $\zeta$ via Rashba spin-orbit coupling. Now here we have a reversed situation.

In terms of possible materials realizations, in 2D materials there are many possibilities. The most prominent example is the organic compound. Replacing the iodine $(\mathrm{I}, \zeta<1)$ by halogens, such as $\mathrm{F}(\zeta>1)$ an average control of $\mathrm{F}$ substitution correspond to a space-dependent tilt [19]. Even a random substitution of halogens can lead to randomness in the $\zeta$ which seems to be an interesting element to randomize and worth investigation, particularly from the point of view of the spacetime structure. Or the interface between I-rich and F-rich compound based on this organic systems can mimic black-hole horizon. ${ }^{8}$ This transition corresponds to crossing a black-hole horizon Furthermore, magnetic textures [85] can also generate space dependet tilt parameter $\zeta\left(x^{i}\right)$.

Therefore, the tilted Dirac cone systems in two space dimensions are promising frameworks for generation of spinorbit coupling (synthetic gauge fields). The effect of spacetime geometry is not limited to such synthetic forces. The quantum emission which is at the heart of solid-state spectroscopies will also be affected by the spacetime curvature [100]. This might require a careful examination of the linear response theory that links theoretical calculations within the Kubo formula with experiments [101].

\section{ACKNOWLEDGMENTS}

We thank Ahmad Reza Moradpour and Armin Ghazi for fruitful discussions and Prof. Dr. Reza Mansouri for insightful discussions and encouragements to push the idea of solid-state spacetime structures. S.A.J. appreciates research deputy of Sharif University of Technology, Grant No. G960214 and Iran Science Elites Foundation (ISEF).

\footnotetext{
${ }^{8}$ One has to be careful that the "effective" description in terms of a metric is valid at length scales much larger than the atomic scales. As such, such solid-state black holes are escapable in the atomic scales [99].
} 


\section{APPENDIX A: DERIVATION OF CHRISTOFFEL SYMBOLS}

In this Appendix we present details of calculations related to the Christoffel symbols defined by,

$$
\Gamma_{\mu \nu}^{\rho}=\frac{1}{2} g^{\rho \sigma}\left(\partial_{\mu} g_{\nu \sigma}+\partial_{\nu} g_{\mu \sigma}-\partial_{\sigma} g_{\mu \nu}\right),
$$

where the metric of the resulting $2+1$-dimensional spacetime with tilt parameters $\zeta=\left(\zeta_{x}, \zeta_{y}\right)$ is given by

$$
\begin{gathered}
g_{\mu \nu}=\left[\begin{array}{ccc}
\zeta^{2}-1 & -\zeta_{x} & -\zeta_{y} \\
-\zeta_{x} & 1 & 0 \\
-\zeta_{y} & 0 & 1
\end{array}\right], \\
g^{\mu \nu}=\left[g_{\mu \nu}\right]^{-1}=\left[\begin{array}{ccc}
-1 & -\zeta_{x} & -\zeta_{y} \\
-\zeta_{x} & 1-\zeta_{x}^{2} & -\zeta_{x} \zeta_{y} \\
-\zeta_{y} & -\zeta_{x} \zeta_{y} & 1-\zeta_{y}^{2}
\end{array}\right]
\end{gathered}
$$

with $\zeta^{2}=\zeta_{x}^{2}+\zeta_{y}^{2}$. The components $\zeta_{x} \equiv \zeta_{1}$ and $\zeta_{y} \equiv \zeta_{2}$ of the tilt are assumed to have arbitrary functional dependence on the space coordinates $(x, y)$ inside the material. Then Christoffel symbols are given by

$$
\begin{aligned}
& \Gamma_{00}^{0}=\zeta_{x} \zeta_{y}\left(\partial_{y} \zeta_{x}+\partial_{x} \zeta_{y}\right)+\zeta_{x}^{2} \partial_{x} \zeta_{x}+\zeta_{y}^{2} \partial_{y} \zeta_{y} \\
& \Gamma_{10}^{0}=\Gamma_{01}^{0}=-\zeta_{x} \partial_{x} \zeta_{x}-\frac{1}{2} \zeta_{y}\left(\partial_{y} \zeta_{x}+\partial_{x} \zeta_{y}\right) \\
& \Gamma_{20}^{0}=\Gamma_{20}^{0}=-\zeta_{y} \partial_{y} \zeta_{y}-\frac{1}{2} \zeta_{x}\left(\partial_{y} \zeta_{x}+\partial_{x} \zeta_{y}\right) \\
& \Gamma_{12}^{0}=\Gamma_{21}^{0}=\frac{1}{2}\left(\partial_{y} \zeta_{x}+\partial_{x} \zeta_{y}\right) \\
& \Gamma_{11}^{0}=\partial_{x} \zeta_{x} \\
& \Gamma_{22}^{0}=\partial_{y} \zeta_{y} \\
& \Gamma_{00}^{1}=\zeta_{x} \zeta_{y}\left(\zeta_{x} \partial_{y} \zeta_{x}+\zeta_{y} \partial_{y} \zeta_{y}\right)+\left(\zeta_{x}^{2}-1\right)\left(\zeta_{x} \partial_{x} \zeta_{x}+\zeta_{y} \partial_{x} \zeta_{y}\right) \\
& \Gamma_{01}^{1}=\Gamma_{10}^{1}=-\frac{1}{2} \zeta_{x}\left(2 \zeta_{x} \partial_{x} \zeta_{x}+\zeta_{y}\left(\partial_{y} \zeta_{x}+\partial_{x} \zeta_{y}\right)\right) \\
& \Gamma_{02}^{1}=\frac{1}{2}\left(\left(1-\zeta_{x}^{2}\right) \partial_{x} \zeta_{y}-\left(\zeta_{x}^{2}+1\right) \partial_{y} \zeta_{x}\right)-\zeta_{x} \zeta_{y} \partial_{y} \zeta_{y} \\
& \Gamma_{21}^{1}=\Gamma_{12}^{1}=\frac{1}{2} \zeta_{x}\left(\partial_{y} \zeta_{x}+\partial_{x} \zeta_{y}\right) \\
& \Gamma_{22}^{1}=\zeta_{x} \partial_{y} \zeta_{y} \\
& \Gamma_{11}^{1}=\zeta_{x} \partial_{x} \zeta_{x} \\
& \Gamma_{00}^{2}=\left(\zeta_{y}^{2}-1\right)\left(\zeta_{x} \partial_{y} \zeta_{x}+\zeta_{y} \partial_{y} \zeta_{y}\right)+\zeta_{x} \zeta_{y}\left(\zeta_{x} \partial_{x} \zeta_{x}+\zeta_{y} \partial_{x} \zeta_{y}\right) \\
& \Gamma_{01}^{2}=\Gamma_{10}^{2}=-\frac{1}{2}\left(\zeta_{y}^{2}-1\right) \partial_{y} \zeta_{x}-\frac{1}{2}\left(\zeta_{y}^{2}+1\right) \partial_{x} \zeta_{y}-\zeta_{x} \zeta_{y} \partial_{x} \zeta_{x} \\
& \Gamma_{02}^{2}=\Gamma_{20}^{2}=-\frac{1}{2} \zeta_{x} \zeta_{y}\left(\partial_{y} \zeta_{x}+\partial_{x} \zeta_{y}\right)-\zeta_{y}^{2} \partial_{y} \zeta_{y} \\
& \Gamma_{21}^{2}=\Gamma_{12}^{2}=\frac{1}{2} \zeta_{y}\left(\partial_{y} \zeta_{x}+\partial_{x} \zeta_{y}\right) \\
& \Gamma_{22}^{2}=\zeta_{y} \partial_{y} \zeta_{y} \\
& \Gamma_{11}^{2}=\zeta_{y} \partial_{x} \zeta_{x} \\
&
\end{aligned}
$$

\section{APPENDIX B: DERIVATION OF THE SPIN CONNECTION}

In an arbitrary geometry, the curvature of spacetime requires to take the derivative of the spinor $\psi$ in a covariant form. This amounts to replacement $\gamma^{\mu} \partial_{\mu} \rightarrow \gamma^{a} e_{a}^{\mu}\left(\partial_{\mu}+\Omega_{\mu}\right)$ where the $\Omega_{\mu}$ is called the "spin" connection, as it needed to take covariant derivative of an "spinor" $[78,88,91,92]$. The frame fields $e_{a}^{\mu}$ will be required to change the basis in a way that a locally flat spacetime is obtained. For a generic manifold defined by metric $g_{\mu \nu}$ with $\mu, v=0,1,2$, there is a locally flat
(Minkowski) manifold defined by metric $\eta_{a b}(a, b=0,1,2)$ tangent to this manifold. Requiring the spacetime length element in both cases to be the same gives,

$$
g_{\mu \nu}=\eta_{a b} e_{\mu}^{a} e_{\nu}^{b} \leftrightarrow g^{\mu \nu}=\eta^{a b} e_{a}^{\mu} e_{b}^{\nu}
$$

which defines the frame fields $e_{\mu}^{a}$. In units of $v_{F}=1$ the tilt metric changes to flat space $\eta_{a b}$ to

$$
\begin{aligned}
d s^{2} & =\left(-1+\zeta^{2}\right) d t^{2}-2 \zeta_{x} d x d t-2 \zeta_{y} d y d t+d x^{2}+d y^{2} \\
& =-d t^{\prime 2}+d u^{2}+d v^{2}
\end{aligned}
$$

which can be obtained by affecting a Galilean boost in Minkowski spacetime:

$$
\begin{aligned}
& x^{\prime 0}=t^{\prime}=t, \\
& x^{\prime 1}=u=x-\zeta_{x} t, \\
& x^{\prime 2}=v=y-\zeta_{y} t .
\end{aligned}
$$

Using the transformation law of tensors,

$$
g_{\mu \nu}=\frac{\partial x^{\prime a}}{\partial x^{\mu}} \frac{\partial x^{\prime b}}{\partial x^{\nu}} \eta_{a b}
$$

and comparing with Eq. (B1) gives the frame fields $e_{\mu}^{a}=\frac{\partial x^{\prime a}}{\partial x^{\mu}}$ as follows:

$$
\begin{aligned}
& e_{0}^{0}=\frac{\partial t^{\prime}}{\partial t}=1, \quad e_{0}^{1}=\frac{\partial u}{\partial t}=-\zeta_{x}, \quad e_{0}^{2}=\frac{\partial v}{\partial t}=-\zeta_{y}, \\
& e_{1}^{0}=\frac{\partial t^{\prime}}{\partial x}=0, \quad e_{1}^{1}=\frac{\partial u}{\partial x}=1, \quad e_{1}^{2}=\frac{\partial v}{\partial x}=0, \\
& e_{2}^{0}=\frac{\partial t^{\prime}}{\partial y}=0, \quad e_{2}^{1}=\frac{\partial u}{\partial y}=0, \quad e_{2}^{2}=\frac{\partial v}{\partial y}=1 .
\end{aligned}
$$

From these frame fields one can then construct,

$$
\omega_{\mu}^{a b}=e_{\lambda}^{a}(x) g^{\lambda \sigma}(x) \nabla_{\mu} e_{\sigma}^{b}(x)
$$

where the covariant derivative of the frame fields are

$$
\nabla_{\mu} e_{\sigma}^{a}=\partial_{\mu} e_{\sigma}^{a}-\Gamma_{\mu \sigma}^{\lambda} e_{\lambda}^{a} .
$$

To construct spin connection we calculate $\omega_{\mu}^{a b}$ as follows:

$$
\begin{aligned}
\omega_{\mu}^{12}= & e_{\lambda}^{1} g^{\lambda \sigma} \nabla_{\mu} e_{\sigma}^{2}=e_{0}^{1} g^{0 \sigma} \nabla_{\mu} e_{\sigma}^{2}+e_{1}^{1} g^{1 \sigma} \nabla_{\mu} e_{\sigma}^{2}+e_{2}^{1} g^{2 \sigma} \nabla_{\mu} e_{\sigma}^{2} \\
= & \left(e_{0}^{1} g^{00}+e_{1}^{1} g^{10}+e_{2}^{1} g^{20}\right) \nabla_{\mu} e_{0}^{2}+\left(e_{0}^{1} g^{01}+e_{1}^{1} g^{11}\right. \\
& \left.+e_{2}^{1} g^{21}\right) \nabla_{\mu} e_{1}^{2}+\left(e_{0}^{1} g^{02}+e_{1}^{1} g^{12}+e_{2}^{1} g^{22}\right) \nabla_{\mu} e_{2}^{2},
\end{aligned}
$$

so that

$$
\begin{aligned}
\omega_{\mu}^{12}= & \left(e_{0}^{1} g^{00}+e_{1}^{1} g^{10}\right) \nabla_{\mu} e_{0}^{2}+\left(e_{0}^{1} g^{01}+e_{1}^{1} g^{11}\right) \nabla_{\mu} e_{1}^{2} \\
& +\left(e_{0}^{1} g^{02}+e_{1}^{1} g^{12}\right) \nabla_{\mu} e_{2}^{2} .
\end{aligned}
$$

Next we have

$$
\begin{aligned}
\nabla_{\mu} e_{0}^{2} & =\partial_{\mu} e_{0}^{2}-\left(\Gamma_{\mu 0}^{0} e_{0}^{2}+\Gamma_{\mu 0}^{2}\right), \nabla_{\mu} e_{1}^{2} \\
& =-\left(\Gamma_{\mu 1}^{0} e_{0}^{2}+\Gamma_{\mu 1}^{2}\right), \nabla_{\mu} e_{2}^{2} \\
& =-\left(\Gamma_{\mu 2}^{0} e_{0}^{2}+\Gamma_{\mu 2}^{2}\right)
\end{aligned}
$$

and furthermore,

$$
\omega_{\mu}^{12}=\left(\zeta_{y} \Gamma_{\mu 1}^{0}-\Gamma_{\mu 1}^{2}\right)=0 .
$$


In the same way we are able to find other components of $\omega_{\mu}^{a b}$,

$$
\begin{aligned}
\omega_{\mu}^{02}= & e_{\lambda}^{0} g^{\lambda \sigma} \nabla_{\mu} e_{\sigma}^{2} \\
= & e_{0}^{0} g^{0 \sigma} \nabla_{\mu} e_{\sigma}^{2}+e_{1}^{0} g^{1 \sigma} \nabla_{\mu} e_{\sigma}^{2}+e_{2}^{0} g^{2 \sigma} \nabla_{\mu} e_{\sigma}^{2} \\
= & \left(e_{0}^{0} g^{00}\right) \nabla_{\mu} e_{0}^{2}+\left(e_{0}^{0} g^{01}\right) \nabla_{\mu} e_{1}^{2}+\left(e_{0}^{0} g^{02}\right) \nabla_{\mu} e_{2}^{2} \\
= & -\nabla_{\mu} e_{0}^{2}-\zeta_{x} \nabla_{\mu} e_{1}^{2}-\zeta_{y} \nabla_{\mu} e_{2}^{2} \\
= & -\partial_{\mu} e_{0}^{2}+\left(-\zeta_{y} \Gamma_{\mu 0}^{0}+\Gamma_{\mu 0}^{2}\right)+\zeta_{x}\left(-\zeta_{y} \Gamma_{\mu 1}^{0}+\Gamma_{\mu 1}^{2}\right) \\
& +\zeta_{y}\left(-\zeta_{y} \Gamma_{\mu 2}^{0}+\Gamma_{\mu 2}^{2}\right) \\
\omega_{\mu}^{01}= & e_{\lambda}^{0} g^{\lambda \sigma} \nabla_{\mu} e_{\sigma}^{1}=e_{0}^{0} g^{0 \sigma} \nabla_{\mu} e_{\sigma}^{1}+e_{1}^{0} g^{1 \sigma} \nabla_{\mu} e_{\sigma}^{1}+e_{2}^{0} g^{2 \sigma} \nabla_{\mu} e_{\sigma}^{1} \\
= & -\nabla_{\mu} e_{0}^{1}-\zeta_{x} \nabla_{\mu} e_{1}^{1}-\zeta_{y} \nabla_{\mu} e_{2}^{1} \\
= & \partial_{\mu} \zeta_{x}+\left(-\zeta_{x} \Gamma_{\mu 0}^{0}+\Gamma_{\mu 0}^{1}\right)+\zeta_{x}\left(-\zeta_{x} \Gamma_{\mu 1}^{0}+\Gamma_{\mu 1}^{1}\right) \\
& +\zeta_{y}\left(-\zeta_{x} \Gamma_{\mu 2}^{0}+\Gamma_{\mu 2}^{1}\right) .
\end{aligned}
$$

Therefore, the nonzero components of the spin connection are eventually given by

$$
\begin{aligned}
\omega_{0}^{02} & =-\frac{1}{2} \zeta_{x}\left(\partial_{x} \zeta_{y}+\partial_{y} \zeta_{x}\right)-\zeta_{y} \partial_{y} \zeta_{y} \\
\omega_{0}^{01} & =-\frac{1}{2} \zeta_{y}\left(\partial_{x} \zeta_{y}+\partial_{y} \zeta_{x}\right)-\zeta_{x} \partial_{x} \zeta_{x} \\
\omega_{x}^{01} & =\partial_{x} \zeta_{x} \\
\omega_{x}^{02} & =\frac{1}{2}\left(\partial_{x} \zeta_{y}+\partial_{y} \zeta_{x}\right) \\
\omega_{y}^{01} & =\frac{1}{2}\left(\partial_{x} \zeta_{y}+\partial_{y} \zeta_{x}\right) \\
\omega_{y}^{02} & =\partial_{y} \zeta_{y}
\end{aligned}
$$

It begs to define the following quantity,

$$
\alpha_{i}^{j}=\frac{1}{2}\left(\partial_{i} \zeta^{j}+\partial_{j} \zeta^{i}\right),
$$

then the compact form of above equations leads to

$$
\omega_{i}^{0 j}=\alpha_{i}^{j}, \omega_{0}^{0 i}=-\alpha_{i}^{j} \zeta_{j}, \text { others }=0 .
$$

Now that we have all the components $\omega_{\mu}^{a b}$ we can contract it with the generators $\Sigma_{a b}=\left[\gamma_{a}, \gamma_{b}\right] / 4$ of the Lorentz group to obtain the spin connection $\Omega_{\mu}=\frac{1}{2} \omega_{\mu}^{a b} \Sigma_{a b}$. As we discussed in the main text of the paper, definition of covariant derivative in the presence of variable $e_{a}^{\mu}$ (variable frame fields) is

$$
\nabla_{a}=e_{a}^{\mu}\left(\partial_{\mu}+\Omega_{\mu}\right)
$$

when the frame fields are not position-dependent, the above equation reduces to

$$
\nabla_{a}=e_{a}^{\mu} \partial_{\mu}
$$

or

$$
\left(\begin{array}{l}
\nabla_{0} \\
\nabla_{1} \\
\nabla_{2}
\end{array}\right)=\left(\begin{array}{ccc}
1 & \zeta_{x} & \zeta_{y} \\
0 & 1 & 0 \\
0 & 0 & 1
\end{array}\right)\left(\begin{array}{l}
\partial_{0} \\
\partial_{1} \\
\partial_{2}
\end{array}\right),
$$

so that the Lagrangian density for a tilted system is

$$
\mathcal{L}=i \bar{\psi}\left(\gamma_{\mu} \partial_{\mu}+\gamma_{0} \vec{\zeta} \cdot \vec{\partial}\right) \psi+c \cdot c,
$$

but for a variable (position-dependent) tilt we get the gauge fields as

$$
\mathcal{A}_{a}=e_{a}^{\mu} \Omega_{\mu} .
$$

This equation establishes that the space dependence of the tilt parameters $\zeta$ in metric Eq. (5), induces non-Abelian gauge fields given by Eq. (16).
[1] A. Zee, Quantum Field Theory in a Nutshell (Princeton University Press, Princeton, NJ, 2010).

[2] M. E. Peskin and D. V. Schroeder, An Introduction To Quantum Field Theory (Avalon Publishing, New York, 1995).

[3] N. Tajima, S. Sugawara, M. Tamura, Y. Nishio, and K. Kajita, J. Phys. Soc. Jpn. 75, 051010 (2006).

[4] S. Katayama, A. Kobayashi, and Y. Suzumura, J. Phys. Soc. Jpn. 75, 054705 (2006).

[5] A. Kobayashi, S. Katayama, Y. Suzumura, and H. Fukuyama, J. Phys. Soc. Jpn. 76, 034711 (2007).

[6] N. Tajima and K. Kajita, Sci. Technol. Adv. Mater. 10, 024308 (2009).

[7] A. Kobayashi, S. Katayama, and Y. Suzumura, Sci. Technol. Adv. Mater. 10, 024309 (2009).

[8] H. Isobe, in Theoretical Study on Correlation Effects in Topological Matter (Springer, Singapore, 2017), pp. 63-81.

[9] K. Kajita, Y. Nishio, N. Tajima, Y. Suzumura, and A. Kobayashi, J. Phys. Soc. Jpn. 83, 072002 (2014).

[10] N. Tajima, T. Yamauchi, T. Yamaguchi, M. Suda, Y. Kawasugi, H. M. Yamamoto, R. Kato, Y. Nishio, and K. Kajita, Phys. Rev. B 88, 075315 (2013).
[11] T. Morinari, T. Himura, and T. Tohyama, J. Phys. Soc. Jpn. 78, 023704 (2009).

[12] S. Sugawara, M. Tamura, N. Tajima, R. Kato, M. Sato, Y. Nishio, and K. Kajita, J. Phys. Soc. Jpn. 79, 113704 (2010).

[13] M. O. Goerbig, J.-N. Fuchs, G. Montambaux, and F. Piéchon, Phys. Rev. B 78, 045415 (2008).

[14] M. O. Goerbig, J.-N. Fuchs, G. Montambaux, and F. Piéchon, EPL 85, 57005 (2009).

[15] J. Sári, C. Tőke, and M. O. Goerbig, Phys. Rev. B 90, 155446 (2014).

[16] M. Hirata, K. Ishikawa, K. Miyagawa, K. Kanoda, and M. Tamura, Phys. Rev. B 84, 125133 (2011).

[17] M. Hirata, K. Ishikawa, G. Matsuno, A. Kobayashi, K. Miyagawa, M. Tamura, C. Berthier, and K. Kanoda, Science 358, 1403 (2017).

[18] M. Hirata, K. Ishikawa, K. Miyagawa, M. Tamura, C. Berthier, D. Basko, A. Kobayashi, G. Matsuno, and K. Kanoda, Nat. Commun. 7, 12666 (2016).

[19] R. M. Geilhufe, B. Commeau, and G. W. Fernando, Phys. Status Solidi RRL 12, 1800081 (2018). 
[20] L. L. Tao and E. Y. Tsymbal, Phys. Rev. B 98, 121102(R) (2018).

[21] C.-K. Chiu, Y.-H. Chan, X. Li, Y. Nohara, and A. P. Schnyder, Phys. Rev. B 95, 035151 (2017).

[22] T. Morinari, E. Kaneshita, and T. Tohyama, Phys. Rev. Lett. 105, 037203 (2010).

[23] A. Varykhalov, D. Marchenko, J. Sánchez-Barriga, E. Golias, O. Rader, and G. Bihlmayer, Phys. Rev. B 95, 245421 (2017).

[24] Y. Imai, F. Nabeshima, D. Nakamura, T. Katase, H. Hiramatsu, H. Hosono, and A. Maeda, J. Phys. Soc. Jpn. 82, 043709 (2013).

[25] H.-Y. Lu, A. S. Cuamba, S.-Y. Lin, L. Hao, R. Wang, H. Li, Y. Y. Zhao, and C. S. Ting, Phys. Rev. B 94, 195423 (2016).

[26] X.-F. Zhou, X. Dong, A. R. Oganov, Q. Zhu, Y. Tian, and H.-T. Wang, Phys. Rev. Lett. 112, 085502 (2014).

[27] A. Lopez-Bezanilla and P. B. Littlewood, Phys. Rev. B 93, 241405(R) (2016).

[28] T. Farajollahpour, Z. Faraei, and S. A. Jafari, Phys. Rev. B 99, 235150 (2019).

[29] T. Kawarabayashi, Y. Hatsugai, T. Morimoto, and H. Aoki, Phys. Rev. B 83, 153414 (2011).

[30] K. Kishigi, K. Ueno, E. Miyamoto, and Y. Hasegawa, Journal of Physics: Conference Series 334, 012047 (2011).

[31] S. A. Herrera and G. G. Naumis, Phys. Rev. B 100, 195420 (2019).

[32] I. Proskurin, M. Ogata, and Y. Suzumura, Phys. Rev. B 91, 195413 (2015).

[33] Y. Suzumura, I. Proskurin, and M. Ogata, J. Phys. Soc. Jpn. 83, 023701 (2014).

[34] S. Rostamzadeh, I. Adagideli, and M. O. Goerbig, Phys. Rev. B 100, 075438 (2019).

[35] S. Verma, A. Mawrie, and T. K. Ghosh, Phys. Rev. B 96, 155418 (2017)

[36] T. Nishine, A. Kobayashi, and Y. Suzumura, J. Phys. Soc. Jpn. 79, 114715 (2010).

[37] T. Nishine, A. Kobayashi, and Y. Suzumura, J. Phys. Soc. Jpn. 80, 114713 (2011).

[38] Z. Jalali-Mola and S. A. Jafari, Phys. Rev. B 98, 195415 (2018).

[39] Z. Jalali-Mola and S. A. Jafari, Phys. Rev. B 98, 235430 (2018).

[40] Y. Gao and D. Xiao, Phys. Rev. B 98, 060402(R) (2018).

[41] D. Sinha, Eur. Phys. J. B 92, 61 (2019).

[42] P. Sengupta, Y. Tan, E. Bellotti, and J. Shi, J. Phys.: Condens. Matter 30, 435701 (2018).

[43] H. Isobe and N. Nagaosa, J. Phys. Soc. Jpn. 81, 113704 (2012).

[44] Z.-K. Yang, J.-R. Wang, and G.-Z. Liu, Phys. Rev. B 98, 195123 (2018).

[45] H.-X. Xiao, J.-R. Wang, H.-T. Feng, P.-L. Yin, and H.-S. Zong, Phys. Rev. B 96, 155114 (2017).

[46] D. Ohki, M. Hirata, T. Tani, K. Kanoda, and A. Kobayashi, arXiv:1910.08999.

[47] Z. Faraei and S. A. Jafari, Phys. Rev. B 100, 245436 (2019).

[48] Z. Faraei and S. A. Jafari, Phys. Rev. B 101, 214508 (2020).

[49] D. Li, B. Rosenstein, B. Y. Shapiro, and I. Shapiro, Phys. Rev. B 95, 094513 (2017).

[50] A. A. Soluyanov, D. Gresch, Z. Wang, Q. Wu, M. Troyer, X. Dai, and B. A. Bernevig, Nature 527, 495 (2015).
[51] F. Y. Bruno, A. Tamai, Q. S. Wu, I. Cucchi, C. Barreteau, A. de la Torre, S. McKeown Walker, S. Riccò, Z. Wang, T. K. Kim, M. Hoesch, M. Shi, N. C. Plumb, E. Giannini, A. A. Soluyanov, and F. Baumberger, Phys. Rev. B 94, 121112(R) (2016).

[52] Y. Wu, D. Mou, N. H. Jo, K. Sun, L. Huang, S. L. Bud'ko, P. C. Canfield, and A. Kaminski, Phys. Rev. B 94, 121113(R) (2016).

[53] C. Wang, Y. Zhang, J. Huang, S. Nie, G. Liu, A. Liang, Y. Zhang, B. Shen, J. Liu, C. Hu, Y. Ding, D. Liu, Y. Hu, S. He, L. Zhao, L. Yu, J. Hu, J. Wei, Z. Mao, Y. Shi, X. Jia, F. Zhang, S. Zhang, F. Yang, Z. Wang, Q. Peng, H. Weng, X. Dai, Z. Fang, Z. Xu, C. Chen, and X. J. Zhou, Phys. Rev. B 94, 241119(R) (2016).

[54] Y. Sun, S.-C. Wu, M. N. Ali, C. Felser, and B. Yan, Phys. Rev. B 92, 161107(R) (2015).

[55] K. Deng, G. Wan, P. Deng, K. Zhang, S. Ding, E. Wang, M. Yan, H. Huang, H. Zhang, Z. Xu, J. Denlinger, A. Fedorov, H. Yang, W. Duan, H. Yao, Y. Wu, S. Fan, H. Zhang, X. Chen, and S. Zhou, Nat. Phys. 12, 1105 (2016).

[56] J. Jiang, Z. K. Liu, Y. Sun, H. F. Yang, C. R. Rajamathi, Y. P. Qi, L. X. Yang, C. Chen, H. Peng, C.-C. Hwang, S. Z. Sun, S.-K. Mo, I. Vobornik, J. Fujii, S. S. P. Parkin, C. Felser, B. H. Yan, and Y. L. Chen, Nat. Commun. 8, 13973 (2017).

[57] L. Huang, T. M. McCormick, M. Ochi, Z. Zhao, M.-T. Suzuki, R. Arita, Y. Wu, D. Mou, H. Cao, J. Yan, N. Trivedi, and A. Kaminski, Nat. Mater. 15, 1155 (2016).

[58] N. Xu, Z. Wang, A. Weber, A. Magrez, P. Bugnon, H. Berger, C. Matt, J. Ma, B. Fu, B. Lv et al., arXiv:1604.02116.

[59] A. Liang, J. Huang, S. Nie, Y. Ding, Q. Gao, C. Hu, S. He, Y. Zhang, C. Wang, B. Shen et al., arXiv:1604.01706.

[60] H. Huang, S. Zhou, and W. Duan, Phys. Rev. B 94, 121117(R) (2016)

[61] K. Zhang, M. Yan, H. Zhang, H. Huang, M. Arita, Z. Sun, W. Duan, Y. Wu, and S. Zhou, Phys. Rev. B 96, 125102 (2017).

[62] F. Fei, X. Bo, R. Wang, B. Wu, J. Jiang, D. Fu, M. Gao, H. Zheng, Y. Chen, X. Wang, H. Bu, F. Song, X. Wan, B. Wang, and G. Wang, Phys. Rev. B 96, 041201(R) (2017).

[63] F. Fei, X. Bo, P. Wang, J. Ying, J. Li, K. Chen, Q. Dai, B. Chen, Z. Sun, M. Zhang, F. Qu, Y. Zhang, Q. Wang, X. Wang, L. Cao, H. Bu, F. Song, X. Wan, and B. Wang, Adv. Mater. 30, 1801556 (2018).

[64] H.-J. Noh, J. Jeong, E.-J. Cho, K. Kim, B. I. Min, and B.-G. Park, Phys. Rev. Lett. 119, 016401 (2017).

[65] M. Yan, H. Huang, K. Zhang, E. Wang, W. Yao, K. Deng, G. Wan, H. Zhang, M. Arita, H. Yang, Z. Sun, H. Yao, Y. Wu, S. Fan, W. Duan, and S. Zhou, Nat. Commun. 8, 257 (2017).

[66] T. Kariyado and M. Ogata, J. Phys. Soc. Jpn. 80, 083704 (2011).

[67] J. F. Khoury, A. J. E. Rettie, M. A. Khan, N. J. Ghimire, I. Robredo, J. E. Pfluger, K. Pal, C. Wolverton, A. Bergara, J. S. Jiang, L. M. Schoop, M. G. Vergniory, J. F. Mitchell, D. Y. Chung, and M. G. Kanatzidis, J. Am. Chem. Soc. 141, 19130 (2019).

[68] M. Trescher, B. Sbierski, P. W. Brouwer, and E. J. Bergholtz, Phys. Rev. B 91, 115135 (2015).

[69] M. Trescher, B. Sbierski, P. W. Brouwer, and E. J. Bergholtz, Phys. Rev. B 95, 045139 (2017).

[70] S. Tchoumakov, M. Civelli, and M. O. Goerbig, Phys. Rev. Lett. 117, 086402 (2016). 
[71] J. P. Carbotte, Phys. Rev. B 94, 165111 (2016).

[72] T. S. Sikkenk and L. Fritz, Phys. Rev. B 96, 155121 (2017).

[73] A. A. Zyuzin and R. P. Tiwari, JETP Lett. 103, 717 (2016).

[74] O. Ar1, I. Dursun, and R. T. Senger, arXiv:1105.0547 (2011).

[75] G. E. Volovik, JETP Lett. 104, 645 (2016).

[76] J. Nissinen and G. E. Volovik, JETP Lett. 105, 447 (2017).

[77] K. Martel and E. Poisson, Am. J. Phys. 69, 476 (2001).

[78] S. Carrol, Spacetime and Geometry: An Introduction to General Relativity (Pearson, London, UK, 2003).

[79] A. Iorio and G. Lambiase, Phys. Rev. D 90, 025006 (2014).

[80] A. Iorio, in Journal of Physics: Conference Series, Vol. 442 (IOP Publishing, Bristol, UK, 2013) p. 012056.

[81] M. Matsuo, J. Ieda, E. Saitoh, and S. Maekawa, Phys. Rev. B 84, 104410 (2011)

[82] M. Matsuo, J. Ieda, E. Saitoh, and S. Maekawa, Phys. Rev. Lett. 106, 076601 (2011).

[83] S. A. Jafari, Phys. Rev. B 100, 045144 (2019).

[84] Z. Jalali-Mola and S. A. Jafari, Phys. Rev. B 100, 075113 (2019).

[85] L. Liang and T. Ojanen, Phys. Rev. Res. 1, 032006 (2019).

[86] A. Westström and T. Ojanen, Phys. Rev. X 7, 041026 (2017).

[87] E. Arias, A. R. Hernández, and C. Lewenkopf, Phys. Rev. B 92, 245110 (2015).
[88] L. Ryder, Introduction to General Relativity (Cambridge University Press, Cambridge, UK, 2009).

[89] T. Fließbach, Allgemeine Relativitätstheorie, Vol. 3 (Springer, Berlin, 2012).

[90] G. B. Arfken and H. J. Weber, Mathematical Methods for Physicists (Elsevier, Amsterdam, 1999).

[91] M. Nakahara, Geometry, Topology and Physics (CRC Press, Boca Raton, FL, 2003).

[92] N. D. Birrell and P. Davies, Quantum Fields in Curved Space (Cambridge University Press, Cambridge, UK, 1984).

[93] M. A. Vozmediano, M. Katsnelson, and F. Guinea, Phys. Rep. 496, 109 (2010).

[94] F. de Juan, J. L. Manes, and M. A. H. Vozmediano, Phys. Rev. B 87, 165131 (2013).

[95] F. Guinea, B. Horovitz, and P. Le Doussal, Solid State Commun. 149, 1140 (2009).

[96] F. Guinea, B. Horovitz, and P. Le Doussal, Phys. Rev. B 77, 205421 (2008).

[97] A. Shitade and E. Minamitani, arXiv:2002.05371 (2020).

[98] T. Stegmann and N. Szpak, 2D Mater. 6, 015024 (2018).

[99] Y. Kedem, E. J. Bergholtz, and F. Wilczek, arXiv:2001.02625 (2020).

[100] F. K. Maxime J Jacquet, arXiv:2001.05807 (2020).

[101] S. M. Girvin and K. Yang, Modern Condensed Matter Physics (Cambridge University Press, Cambridge, UK, 2019). 